\title{
Functionality and performance of the ALFA_CTPIN module
}

\section{Wieslaw Iwanski ${ }^{\mathbf{1}}$}

Innsbruck University

CERN, CH-1211, Geneva, Switzerland

E-mail: wieslaw.iwanski@cern.ch

\section{Sune Jakobsen}

CERN

CH-1211, Geneva, Switzerland

E-mail: sune.jakobsen@cern. ch

\section{Krzysztof Korcyl}

Institute of Nuclear Physics Polish Academy of Sciences

Radzikowskiego 152, 31-342 Krakow, Poland

E-mail: krzysztof.korcyl@ifj.edu.pl

\section{Jan Oechsle}

University of Copenhagen

Blegdamsvej 17, 2100 Copenhagen, Denmark

E-mail: jan.oechsle@nbi.dk

The ALFA_CTPIN module has been designed in response to increased internal processing time of the Central Trigger Processor (CTP) of the ATLAS experiment which resulted in reducing time left to the ALFA detector to deliver its own triggers to the CTP within specified latency. Accelerated extraction of ALFA triggers from encoded signals and the possibility to perform local triggers processing by this module allowed ALFA to contribute to global triggering of the ATLAS detector. A huge number of implemented scalers and flexibility in defining triggers processing criteria make also from this module a very attractive tool for in-depth analysis of properties of the LHC beam.

Topical Workshop on Electronics for Particle Physics

11 - 14 September 2017

Santa Cruz, California

\section{${ }^{1}$ Speaker}




\section{Introduction}

During the first Long Shutdown (LS1) of the Large Hadron Collider (LHC), the Central Trigger Processor (CTP) of the ATLAS experiment has been upgraded [1]. In addition to enriched functionality, it resulted in increasing the overall CTP input-output latency by 75 ns ( 3 cycles@ 40 MHz). The ALFA triggers [2] were no longer early enough to contribute to the global ATLAS triggering, which could compromise the physics' program. A dedicated input board, speeding up processing and providing advanced monitoring of the ALFA trigger signals, has therefore been required.

\section{General description}

The ALFA_CTPIN board has been designed in the VME standard. On its inputs, the module receives the LHC Bunch-Crossing (BC) and Orbit signals [3] from the ATLAS CTP and up to 16 trigger signals from the ALFA detector. After a short local processing, the ALFA triggers are then driven to the ATLAS CTP.

The whole module's functionality has been implemented in two FPGA chips. The block diagram presenting the module's logic consisting of the MD/OD Triggers Separator, Phase Shift Measurement unit, Triggers Processing unit, Simple Scalers, BC Scalers, Triggers Patterns Generator and VME Interface is shown in Figure 1.

The primary job of the module is to extract ALFA detector triggers from encoded input signals and send them to the CTP. This time critical data path is shown in red colour in the module's block diagram. An alternative path of the trigger information flow through the Triggers Processing unit (dotted yellow line) takes 50 ns longer ( 2 cycles@ $40 \mathrm{MHz}$ clock). The rest of the module's functionality allows for advanced monitoring of the detector's trigger information.

\section{Functional blocks}

\subsection{MD/OD Triggers Separator}

The MD/OD Trigger Separator unit processes input signals coming from the ALFA detector stations. Each detector station sends to the ALFA_CTPIN module encoded trigger signals coming either from the Main Detector (MD, $25 \mathrm{~ns}$ long pulse) or from the Overlap Detector (OD, $12.5 \mathrm{~ns}$ long pulse). The MD/OD Trigger Separator detects the trigger's type from input signals and produces on its outputs unique, $\mathrm{BC}$ aligned, $\mathrm{MD}$ or $\mathrm{OD}$ trigger pulses driven directly to the ATLAS CTP module.

The maximum latency of the trigger detection is 50 ns (4 cycles@80 MHz) while the 75 ns dead-time of the overall detection process is still inside $100 \mathrm{~ns}$ dead-time of the ALFA detector itself.

\subsection{Triggers Processing}

Apart from being driven directly to the ATLAS CTP, the MD and OD triggers are also made available to the Triggers Processing unit for further processing. For processing of trigger information, the module uses RAM, which offers large flexibility in defining trigger items. Other words, it allows for easy and dynamic change of physics combination to look for and which 
bunches to focus on. The block diagram of this part, consisting of the Mask and Look-Up-Table (LUT) memories, combinatorial cells and the Function selector, is shown in Figure 2.

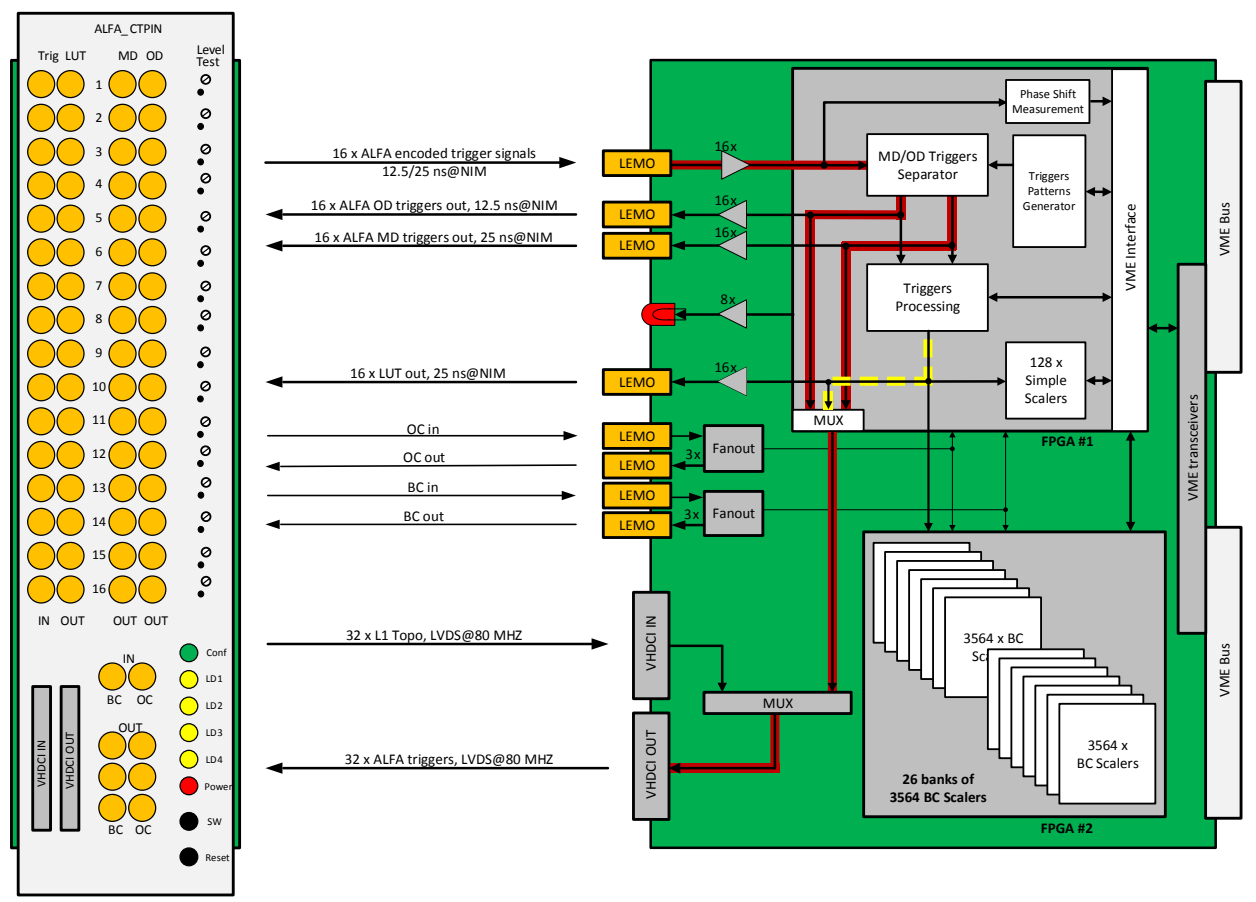

Figure 1: Block diagram of the ALFA_CTPIN module

The MD and OD triggers address individual MD and OD LUTs. The outputs from LUTs then pass through a combinatorial cells stage producing 128 processed trigger items on the unit's output. The four predefined AND/OR/NOT operations performed by combinatorial cells are described in the picture's corner. Although the Function is individual for each processed bit, its value is static for the whole data run. The Mask memory allows for applying individual inhibit to any bit of output data for any bunch number within one Orbit signal. Contents of the Mask, LUT memories and the Function selector is pre-settable via VME. The input-output latency of the Trigger Processing unit is 25 ns (1 cycle@40 MHz).

\subsection{Scalers}

The 128-bit wide data leaving the Triggers Processing unit feed the Scalers stage for calculating rates of processed trigger items. There are two types of scalers: the Simple Scalers and the BC Scalers.

The Simple Scalers sum up the trigger items' count over unit of time on each bit of 128-bit data coming from the Triggers Processing unit. There are therefore 128 of them in the ALFA_CTPIN module. The unit of time is selectable and can be either 1 or 10 seconds.

The BC Scalers sum up their counts occurring for each BC number over unit of time. Here again, the unit of time is selectable and can be either 1 or 10 seconds. As 3564 scalers are needed to cover all possible BC numbers for one trigger item [3], their implementation was limited to 26 trigger items. This ended up with nearly 100000 BC Scalers implemented in FPGA \#2 of the ALFA_CTPIN. Process of updating scalers' count value for a given trigger item takes $62.5 \mathrm{~ns}$ and during that time processing of new hits on this trigger item is not possible. This dead-time however is still inside the dead time of the ALFA detector. 


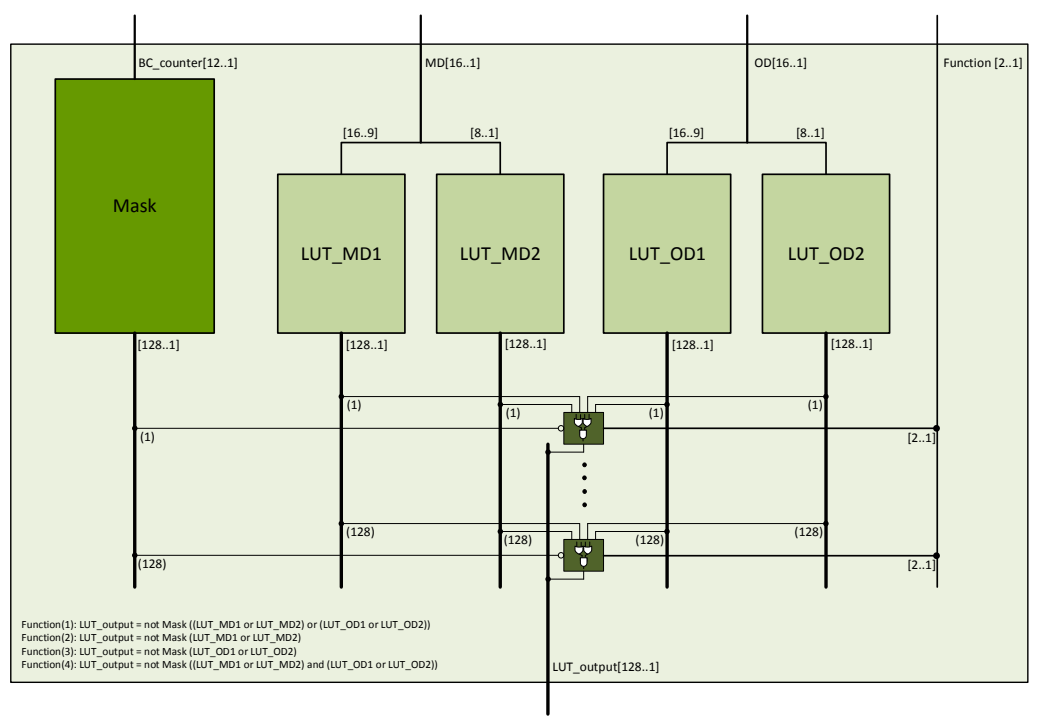

Figure 2: Block diagram of the Triggers Processing unit

\subsection{Phase Shift Measurements}

The task of the Phase Shift Measurement Unit is to provide a coarse information about phase of input signals with respect to the main clock of the ALFA_CTPIN module. The resolution of the measurements is $2.5 \mathrm{~ns}$, the results are made available via VME registers. The unit posts also alerts in case of loss of input signals.

\subsection{Triggers Patterns Generator}

The Triggers Patterns Generator is very strong tool for testing, validating and commissioning of the whole design. It allows for generation of any patterns of encoded trigger signals which may arrive to inputs of the ALFA_CTPIN module from the ALFA detector stations within one Orbit signal of the LHC. The test triggers are programmable, are stored in 8192 deep memory and ran with $80 \mathrm{MHz}$ clock to be able to generate either $12.5 \mathrm{~ns}$ (OD) or $25 \mathrm{~ns}$ (MD) long encoded trigger signals. Once enabled, they are injected directly to the input stage of the ALFA_CTPIN module, then they travel throughout the logic as the real triggers do.

\section{Software}

A dedicated, standalone software has been developed to create and verify the logic for trigger items. The configuration of the module and access to rates of the monitored trigger items uses ATLAS TDAQ software. The ALFA_CTPIN module is configured and controlled by the RCD application. Every $1.3 \mathrm{sec}$ the scalers from the module are read-out using the VME interface. The collected data are then published either as raw numbers or in form of histograms presenting time evolution of monitored items.

\section{Module's application}

Flexibility in defining triggers processing algorithm allows for using the module in large palettes of applications. One of them i.e. analysis of the LHC beam properties is presented here. The histograms shown in Figure 3 were collected during ALFA special run no. 309039 in autumn 2016 with protons in 5 colliding bunches no. 11, 714, 1247, 1875 and 2430. They represent trigger 
rates in the ALFA detectors after 14 hours of running. While the top left histogram shows distribution of protons in adjacent bunches which generate signals in the ALFA detectors, the bottom left one shows the same phenomena but zoomed in around bunch 714 . The top right histogram compared to the top left indicates asymmetry in filling the two beams of the LHC. And finally, the bottom right one illustrates performance of the module in measuring total rates of trigger items coming from 16 ALFA detector stations.

In all histograms, the $\mathrm{Y}$ axes are scaled in numbers of readouts.

No other instrument at LHC can measure beam's properties that way. Further analysis of this data will hopefully bring much knowledge about the LHC parameters like de-bunching speed, pattern of de-bunching after e.g. collimator movement, recapture of particles and many others.
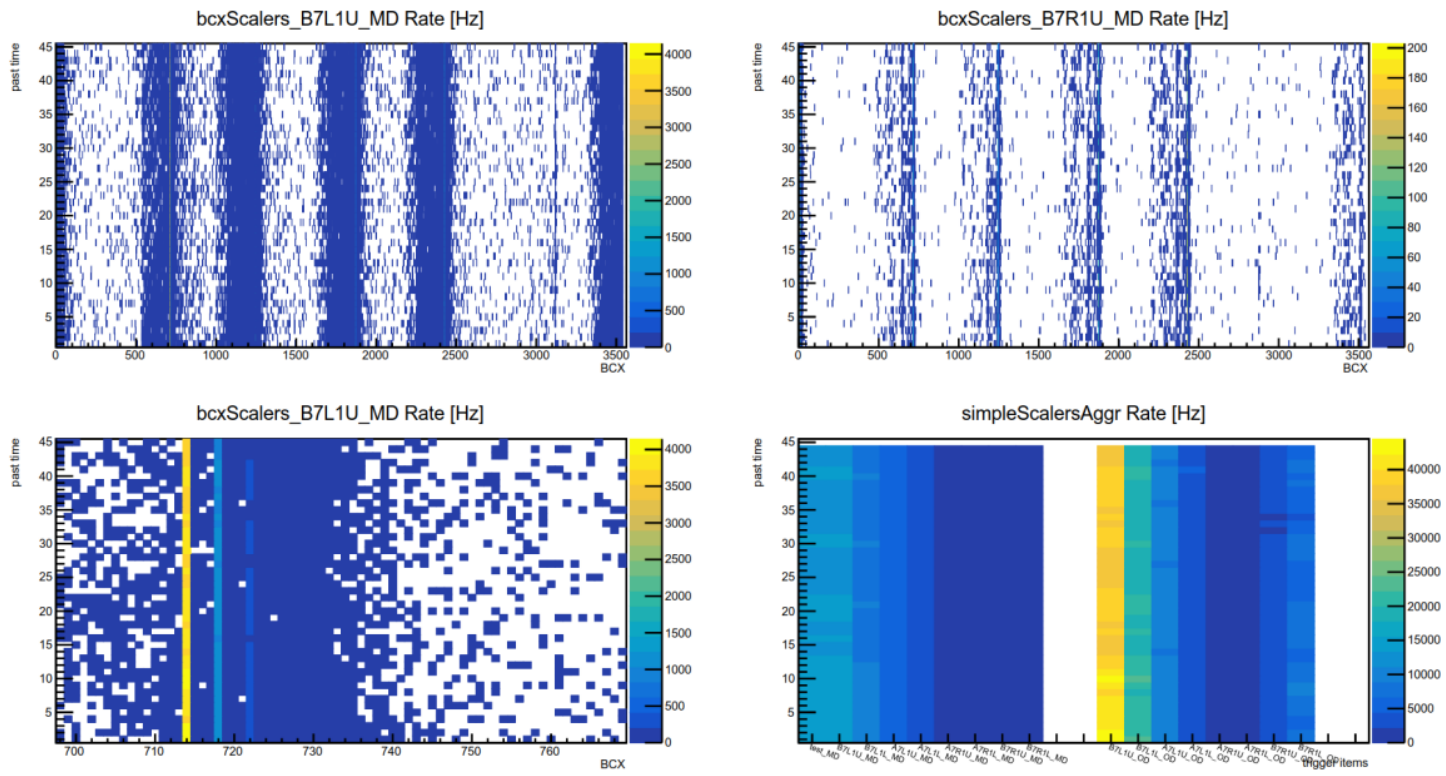

Figure 3: ALFA triggers in special run with protons in 5 bunches: 11, 714, 1247, 1875 and 2430 at LHC

\section{Conclusions}

The ALFA_CTPIN module has been designed in response to increased overall input-output latency of the ATLAS CTP. The module took successfully part in the data taking campaign during Run-2 period and allowed the ALFA detector to be still integrated with triggering process of the ATLAS experiment. The local triggers processing and its flexibility in defining the processing's algorithm as well as giant number of scalers available in the module brought added value and allowed for using the module in precise analysis of the ALFA trigger items as well as in studying the LHC beam properties with single BC level precision.

\section{References}

[1] H. Bertelsen et al, Operation of the upgraded ATLAS Central Trigger Processor during the LHC Run 2, 2016 JINST 11 C02020.

[2] S. Abdel Khalek et al, The ALFA Roman Pot detectors of ATLAS, 2016 JINST 11 P11013.

[3] B. Taylor, Timing distribution at the LHC, in proceedings of The LECC 2002 Workshop, Colmar France, http://cdsweb.cern.ch/record/592719 